\title{
Research on the Wireless Sensor Network Management Methodologies based on the Runtime Model and Game Theory
}

\author{
Wenzhun Huang and Shanwen Zhang \\ Department of Electronic Information Engineering, Xijing University, \\ $X i^{\prime}$ an 710123, China \\ huangwenzhun@xijing.edu.cn;wjdw716@163.com
}

\begin{abstract}
In this paper, we conduct research on the wireless sensor network management methods based on the runtime model. With the deepening of the research, scalability and maintainability of wireless sensor network has become an important target of its application promotion. Consider that the nodes randomly distributed monitoring area, looking for a complete coverage of this area several disjoint nodes which uses genetic algorithm to optimize the network survival time nodes and corresponding coverage. From the point of view of software engineering, most of the specific software system knowledge hidden in the program and document, the model as the main content of the document and procedures of high-level abstractions. The management of the network is urgently needed. As the additional research, we also conduct theoretical analysis on the wireless sensor network security enhancement methodology with the tradition game theory and mathematical optimization approaches which will be meaningful. Game theory is on the interaction between much of decision-making behavior has, according to the different subjects in the control information and the cognition of their own capabilities which will be a novel method for the analysis. The numerical simulation shows that our method performed better compared with other related approaches. In the future, more research will be conducted to polish the current method.
\end{abstract}

Keywords: Wireless Sensor Network, Runtime Model, Cloud Environment; Numerical Analysis, Wireless Communication and Storage, Data Collection, Game Theory

\section{Introduction}

Wireless sensor network is a distributed sensing information system, composed of spatial distribution and collaborate more nodes. These nodes have very limited computation, wireless communication and storage space and energy. With the deepening of the research, scalability and maintainability of wireless sensor network has become an important target of its application promotion. The wireless sensor network has the characteristic such as limited resources and the diversity of hardware and application, makes wireless sensor network application development and deployment are difficult. This requires developing the middleware architecture, providing appropriate abstraction layer to hide operating systems and hardware technical details, under the condition of the limited energy resources to deploy applications to the wireless sensor network so quickly. Wireless sensor network is composed of a ubiquitous and the tiny sensor nodes with wireless communication and computing ability of self-organizing distributed communication network system, more is to be able to finish the task given their own according to environment intelligent collaborative system. Wireless sensor network to realize the high fidelity of a large number of distributed space-time data sampling, but in general applications of sampling frequency of sensor nodes is usually fixed, and the adaptive sampling frequency sampling technique, the changes according to the measured 
object, when the observation object changes slowly lower sampling frequency, when the observation object changes rapidly increase the sampling frequency [1-3].

At present, the wireless sensor network survival time optimization algorithm mainly includes: static survival time of wireless sensor network optimization algorithm and has a single mobile nodes of wireless sensor network survival time optimization algorithm. Consider that the nodes randomly distributed monitoring area, looking for a complete coverage of this area several disjoint nodes, use genetic algorithm to optimize the network survival time nodes and corresponding coverage. Data collected by the sensing device is in real time, large number and without good structure, to apply mapped to the data collected in the scene object attributes, objective things had to write a great deal of conversion code, facing two main challenges. On the one hand is the diversity of the device type, there are different types of sensor devices on the different information such as sound, light, location, monitoring, and different types, different brand, even different types of sensor devices often provide different ways of data read, therefore, the diversity of the device type has brought information gain greater complexity. On the other hand is a management services on demand, there are different types of applications [4-6]. IoT system is actually in the objective world of all kinds of information collection, analysis and decision making process, from the perspective of system implementation, the application system need to use the sensing device provides the management interface for all kinds of information, and in view of the specific application scenario for the information analysis and processing [7].

IoT system development the main problem is: the problem domain and the system implementation, there is a gap between the problem domains is achieved by hard coded into the system to realize mapping will bring huge programming complexity. The software architecture with a set of manageable unit to represent the overall architecture of the system, and can play a bridge between the system requirements and system, is used to solve the demand to realize the process of mapping problem brought by the complexity of system [8]. From the point of view of software engineering, most of the specific software system knowledge hidden in the program and document, the model as the main content of the document and procedures of high-level abstractions, is the ideal software and the carrier of knowledge management. The runtime software architecture model with a set of manageable unit to represent the overall architecture of the system, and through will hide in the system of internal structure, status, configuration and runtime information display to describe as a standard, management perspective oriented structural view, can effectively improve the development of IoT systems of abstraction and automation. The runtime software architecture model has won the wide attention in academia and industry. A considerable amount of research work proved it under different system and management mode of important role. In the Figure 1, we show the general topology of WSN.

In this paper, we conduct research on the wireless sensor network management methodologies based on the runtime model. Cluster head nodes according to the history of the sensory data model and the current sampling data of a sentence, whether according to meet the demand of precision of sampling. If the sensor sampling data within a cluster of dynamics is lower, the lower sampling frequency in order to reduce the redundant data generation and transmission, if the sensor of the dynamic characteristics of the sampling data is higher, then improve the data sampling frequency to ensure the quality of data sampling, which realizes the network energy efficient and the balance between the two data acquisition quality. In the following sections, we will discuss the issues in detail. 

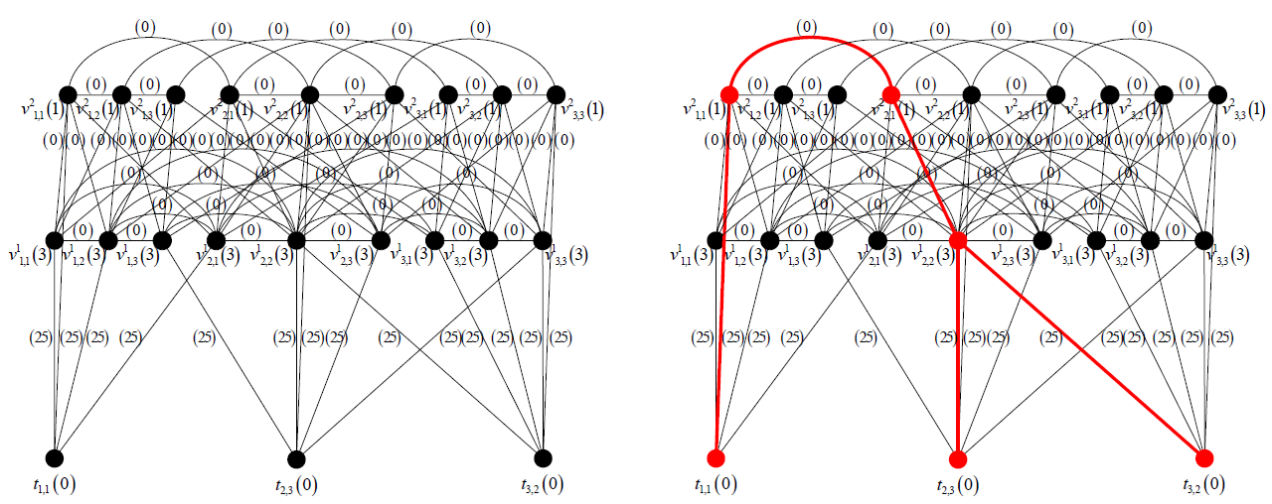

Figure 1. The General Topology of the Wireless Sensor Network

\section{Our Proposed Methodology and Approach}

\subsection{The Runtime Model and Cloud Environment}

Cloud resources run time model is generally practical hardware and software system of abstract in cloud environment, the construction method of input including system model and access model, among them and the model describes the managed information system, access model describes the management of the call interface methods. Cloud management is, in fact, the cloud resource management, and different management requirements of target managed resources also each is not identical [9]. Thus, we put forward the combined methods of distributed runtime model, runtime model in cloud resources, on the basis of in the form of a portfolio model to the organization, implements a variety of cloud resources unified management, the main work includes model customization and data synchronization.

Dynamically adjust processor core resources between different application load and its internal distribution and management. Centralized performance for processor cores by resources unified controller that is allocated to the runtime environment, to ensure the fairness and effectiveness of resource allocation, and processor core resources independently by the runtime environment, distributed management and scheduling, ensure the flexibility of resource use. Advantage is fully embodies the multi-core programming model good programmability and portability, eliminates the traditional multicore runtime environment explicitly specify the number of nuclear, enhances the processor core resource allocation efficiency and adaptability. Currently supports multicore programming model of runtime environment are independent of each other, each other which cannot communication when a variety of mixed programming model, overall system performance optimization, and the current operating system also lack for different multi-core programming model performance optimization method of the runtime environment makes the new programming model in the larger performance bottlenecks in practical application [10].

Customized model pieces need to be able to run from the cloud resource model for the managed resource information, namely the identification of model fragments. Cloud resources run time model and the user customization model fragment is stored in the form of an XML file, for each element in the model, has one and only one path from the root node can locate to the element. The combination model of model fragments from different cloud resource runtime model, they are not grammatical correlation. Therefore, we will each root node of the model fragment as the combination model of the root node direct child nodes. At the same time, in order to solve the model fragment naming conflicts, we also need to maintain a namespace and name to replace the conflict and record. Inside the portfolio model, named element using the new will not conflict, and when the combination model and the runtime model interaction occurs, was named 
element is changed according to the namespace to restore original name that can be run time model identification. In the model on the basis of custom, only maintain combination model and the system is running model of data synchronization, to achieve the unity of the combination model of multiple systems management. Portfolio model made of different elements of the system is running model, the data consistency by the different model fragments were completed. Model fragment operations through the model of transmission and perform to implement the data synchronization [11-15]. On the one hand, when the administrator to manipulate, the portfolio model its model of operation will be sent to the system is running model and implementation. Runtime model in the system, on the other hand, the deployment of a copy of the model fragment, by polling mechanism is found that the change of the model, to automatically generate the corresponding model, sent to the portfolio model and executed. In the Figure 2, we demonstrate the basic concept of the runtime model.

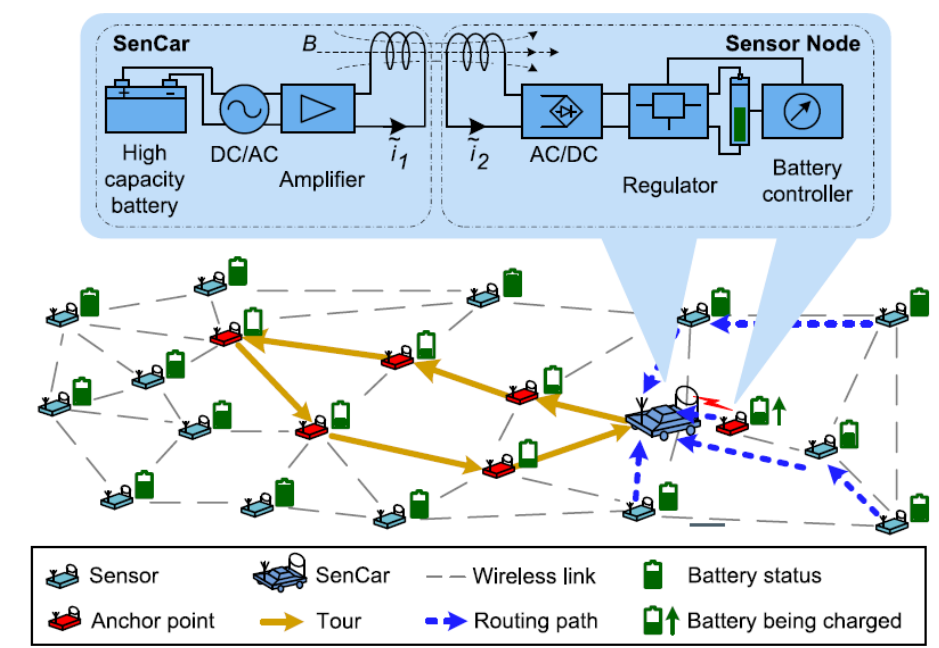

Figure 2. The General Demonstration of the Runtime Model

\subsection{The Wireless Sensor Network Management}

Clustering network structure has the feature of good expansibility, can provide convenient energy management mechanism, the load balancing, resource allocation and data fusion processing all have good performance, such as for unicast, multicast, and broadcast communication is very efficient. Wireless sensor network is different from the Ad hoc network, the node after setting, generally less mobile, so there is no Ad hoc based network due to node mobility of the network topology changes frequently [16-19]. For this kind of "quasi static network, frequent for tech-oriented of clustering is not necessary, therefore, in the initial stages of clustering nodes try to collect the perimeter of the network information, and make a local optimal choice, accordingly in order to realize more stable clustering structure, reduce tech-oriented to the number of clustering, in the early to do so more information interaction, but to prolong the network life cycle is beneficial. The node structure is shown in the Figure 3. 


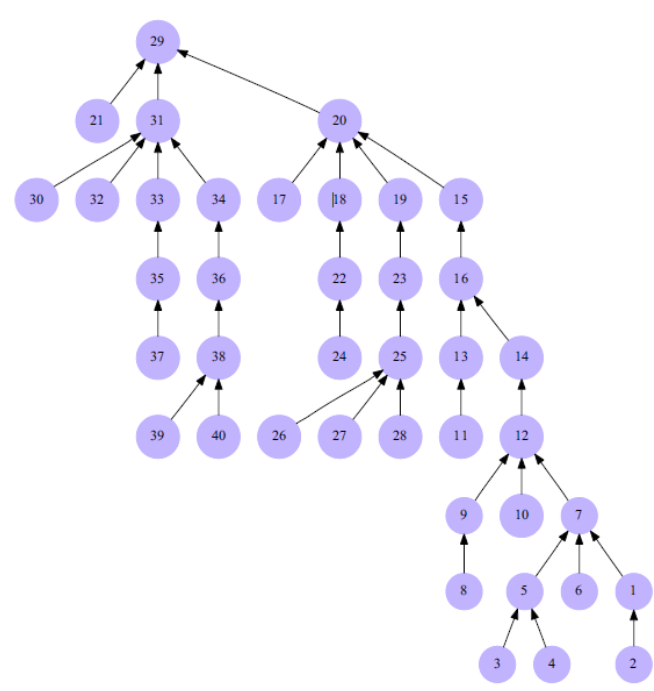

Figure 3. The Node Structure of the Wireless Sensor Network

For perception and gathering information about network coverage range detection object, and jump for short distance wireless communication mode to analysis and processing monitoring data sent to the gateway which is shown in the following formula.

$$
I(x, y, z, t)=\left.[\mathrm{S} 1, \mathrm{~S} 2, \ldots, \mathrm{Sm}]^{\prime}\right|_{(x, y, z, t)}
$$

In the process of the data transmission of wireless sensor network, transmission distance, interference and multipath fading factor will decrease the rate of the success of package receiving. Studies have given the following conclusion: in noise power and the packet length must be a certain circumstances, the closer from the sending node of the corresponding received signal power is stronger, the signal-to-noise ratio, the greater the package to receive the higher the rate of success. Considering the influence of various negative factors, package receiving rate of success is not entirely decreases with the increase of the distance it also has some unexpected.

Because of the wireless sensor node microprocessor under homogeneous configuration need management under the module more than heterogeneous configuration scheme of management module, and the need to deal with protocol communication task than heterogeneous manner to deal with protocol communication task, so it can deduce the microprocessor in the wireless sensor node from heterogeneous isomorphism under the configuration and the related scheme of work time under the configuration scheme of work time [20].

$$
y=\sum_{x \in \Theta} a_{x} h_{x}\left(\theta+\eta_{x}\right)+v
$$

Under the wireless sensor node in homogeneous configuration scheme adopts star network cluster method, each sensor node of sensory information through the cluster head nodes will be sent to the rear database for data fusion. According to the save energy of nodes and prolong survival time of the timing system polling way up [21]. Data correlation clustering mechanism data correlation is the main train of thought of the strong $\mathrm{f}$ embodied in physical space position and average data is relatively close to the sensor nodes in a cluster, each sensor node in a cluster of centralized data model predicts that the cluster head node is responsible for maintaining data in each cluster model to reduce the redundancy of data, improve the efficiency of energy use. Most of the time be monitored environment generally do not have events; If events, events will continue for a period of time, generally within the sliding window other moments will detect the occurrence of the event. Sensor nodes in a certain period of time the readings will be 
related to each other so should make full use of the node in different time to collect data effectively for the event to determine [22].

Sensor node energy limited is one of the most important characters of wireless sensor network system, and the time-varying multipath fading characteristics of wireless channel is one of the main factors that cause the loss of network node energy efficiency. At close range if the clustering process, several nodes by some mechanism into a cluster, and will be near to transfer data within a cluster node and its partner node organized to work together, multiple single antenna nodes to form a virtual multi-antenna system. The power dissipation at the transmitter can be modeled as:

$$
p_{t}(i, k)=c_{i k} \cdot f_{i k}
$$

Sensor node is divided into several clusters, each cluster consists of members of the cluster head nodes and node, the principle of clustering is adjacent sensor nodes collect data correlation is high and the need to the cluster head is responsible for the receiving, fusion and forward data within clusters. Within the cluster member nodes are responsible for perception, collect data, and the detected data is sent to the cluster of cluster head nodes. The following formulas show the optimization steps.

$$
\begin{gathered}
V_{i B}+\sum_{k \leq M+N}^{k \neq i} V_{i k}-\sum_{m \leq M+N}^{m \neq i} V_{i m}-g_{i} T=0 \\
\sum_{i=1}^{N+M} \lambda_{i}=M \quad \sum_{i=1}^{N+M} \mu_{i}=1
\end{gathered}
$$

Within each cluster is generally data fusion of cluster head nodes, is responsible for receiving data message from the cluster member nodes after data fusion to organize and manage clusters. For large-scale wireless sensor network, network topology structure based on clustering in topology management, energy efficiency, data fusion and coordination treatment has advantage. But the basic operating mode are obviously different from the traditional wireless sensor network computer network, sensor network is a distributed environment, each sensor node is an embedded system platform, sensory data, data calculation and the ability to store data, but compared with the traditional computer hardware resources and extremely limited. In addition, the application of sensor networks is closely related to the usage scenario, this makes the traditional middleware development technology cannot be directly applied to sensor network [23]. Cluster head can receive multiple sensor information fusion and intelligent processing accordingly, containing low complexity of detection algorithm, and to detect the signal is sent to the receiver. To deal with the issues, optimization work should be conducted and adopted to modify the traditional methods. In the Figure 4, we show the receiver and caster organization in the wireless sensor network for optimization.

Wireless sensor network is often made up of many different types of sensors and the application system needs to be collected according to different sensing device management goal of the unified analysis and processing of data. For example, the security system needs to monitor area all location-aware equipment, and energy saving system needs in the region to monitor all lighting equipment and brightness perception. These sensing devices need to be unified management, in order to realize the different types of complex management tasks. This paper proposes a distributed runtime model merging method, on the basis of the sensing device runtime model, in the form of a portfolio model to the organization, realize the unified management of different kinds of sensing device as the main work includes model customization and data synchronization. The basic Assumption for the system is shown as the follows. (1) When a sensor node is beyond the scope of the Sink node jump to collect more, all sensor nodes of sensory data in the cache first, and basic in the sleep state. When a sensor node within the scope of the 
Sink node jump to collect more, sensor nodes from sleep wake up for work status, will cache data sent to the Sink node. (2) Only consider 2D terrestrial wireless sensor network and underwater wireless sensor network. Sensor node position is fixed, but can be moved to the Sink node monitoring area to collect data on any position. (3) To avoid moving route choice since the appeared in the process of circulation, the Sink node in the initial position or an anchor point after moving into the center of its neighbor grid, to return to its original position. (4) Multiple exists in the wireless sensor network in a route circulation mobile Sink node. During the process of static and mobile sink node data gathered around the sensor nodes. (5) All the nodes can be used by GPS module or RSSI positioning position method so as to obtain its own position coordinates.

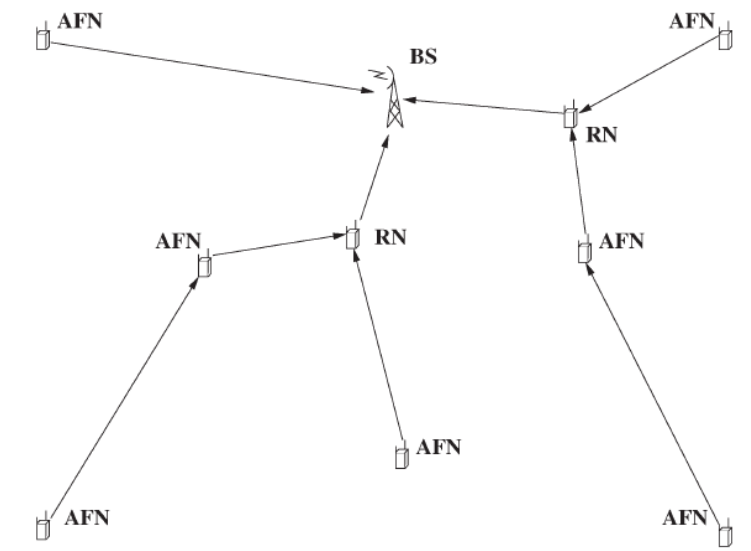

\section{Figure 4. The Receiver and Caster Organization in the WSN for Optimization}

Combination model of model fragments from different sensing device runtime model, they are not grammatical correlation [24]. Therefore, we will root node of the segments of each model as a portfolio model direct child nodes of the root node merging. At the same time, in order to solve the element naming conflicts between each model fragments, we also need to maintain a namespace, to replace conflict element naming and record. In the Figure 5, we use the diagram to describe the proposed wireless sensor network management methodology.

Event boundary nodes on both sides of the edge of the area are distributed in the event, in fact, the node data thus collected were divided into two classes. The cloud resource runtime software and hardware system of abstract model is a cloud environment. The administrator only managed modules to the system of information and system management of the call interface method is described, the construction method can generate the corresponding runtime model cloud resources, and support the runtime model with the state of the system automatic synchronization. So, the administrator can manage the cloud resources in the model layer. Event boundary detection algorithm based on the classification principle of design, when within the neighborhood of a node can be collected at one point the data is divided into two class, is the node can be judged event boundary nodes. Events and event boundary detection algorithm with fault-tolerance ability at the beginning of the sensor nodes to collect the data of the initial stage, the first to perform fault detection algorithm if found node event boundary conditions, the decision for the event boundary nodes, or to determine the fault node, and the execution node information reliability recovery mechanism if convicted for normal nodes, then continue to execute event boundary detection algorithm and the node information reliability recovery mechanism. No matter what kind of results, finally for node will continue to collect data, and perform the incident detection algorithm. Therefore, in this work we present a performance analysis of distributed estimation in WSN employing a 
stationary based framework to characterize the stochastic geometry of such networks. Since, it is based on a random analysis of WSN and it has a good practical utility as it can be used in scenarios with irregular placement of sensor nodes. In the next section, we will test our proposed method with more other state-of-the-art methodologies.

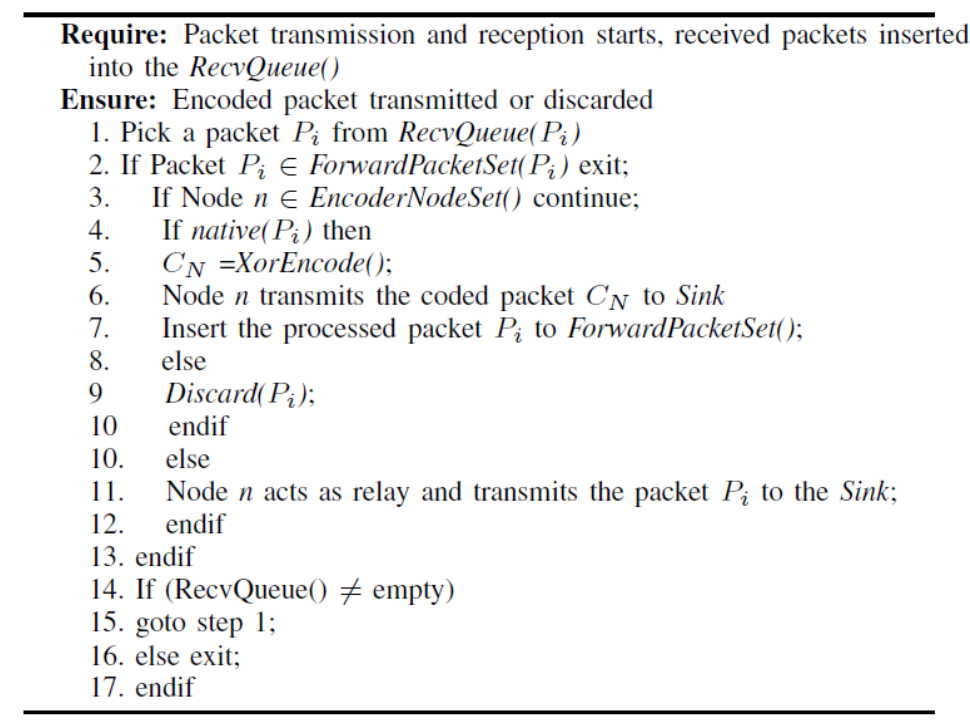

\section{Figure 5. The Algorithm of the Proposed WSN Management Methodology}

\subsection{The Wireless Sensor Network Security and Robustness Enhancement}

Wireless sensor network is often used in unattended unsafe area, so need to focus on security issues. However, sensor nodes resources and computation ability is limited, so compared with the traditional computer networks, wireless sensor network security problems faced with new challenges. At present most of the wireless sensor network security research mainly focuses on the efficient encryption algorithm, the physical security protocols against attack on the data link layer, network layer routing security protocol and application layer and security multicast key management. These security measures can effectively improve the security of the system, but still cannot avoid the network information in the process of space transmission was illegal to intercept [25-26]. In our research, we adopt the idea of game theory to optimize the network structure to enhance the robustness.

Game theory is on the interaction between much of decision-making behavior has, according to the different subjects in the control information and the cognition of their own capabilities, make the correct decision of an action. Currently, the main research on intrusion detection and most based on a one-off game analysis, and in view of the real scene attack could not be launched an attack, only considering the repeat attack, attack the malicious nodes in wireless sensor network as a repeat of the process of network attack multi-stage game to study clearly is more reasonable. Although the form of repeated game is repeated game, but the game behavior and game party profits but it's not a simple repetition, simple stage game party for the interests of the future importance makes them change their appreciation of the interests, which affect their choice strategy in the process of repeated game plan. This means that this article is not the profit that repeated game as stage game yields simple addition, have to put the whole game process as a whole. The overall performance is shown below.

$$
u=u_{1}+u_{2} \varsigma+u_{3} \varsigma^{2}+\ldots+u_{t} \varsigma^{t-1}
$$


In game theory, Nash equilibrium is the fundamental solution of cooperative game, has the stability of the Nash equilibrium is to discover the strategy of portfolio, regardless of whether or not the only solution, there is a common feature, is each of the participants in the game strategy is aimed at the other side of the game participants is the optimal strategy. Through the analysis of the game model between the attacker and IDS achieve Nash equilibrium of the IDS uses a defense strategy [27-28]. If IDS to protect the variety of the attacker is trying to attack, then the attack is failure, the IDS will make a great deal of profit and if the attackers cluster is not cluster IDS protection, then the attacker will make a lot of profit. Observed by the classic game model, although can detect attacks, there is insufficient. First of all, the process of the game on both sides of the game may not be a one-off game, the game is repeated many times, often the result of the game and the result is not the same in a one-off game, not a one-off game enough game strategy, less predictable, so this article chooses repeated game as a basis to examine the invaders and intrusion detection system. Secondly, IDS can detect attack probability is not all to be one, the IDS detection rate will also affect the outcome of the intrusion detection rate is high, then the probability of attack was detected, the smaller the impact on the network also, so can consider joining the intrusion detection system can detect attacks the probability is analyzed. The protection model could be formulated into the following equations.

$$
\begin{gathered}
R D M=\left\{\theta_{i}, \theta_{j} ; A_{i}\left(\theta_{i}\right), A_{j}\left(\theta_{j}\right) ; u_{i}\left(\theta_{i}\right), u_{j}\left(\theta_{j}\right) ; T\right\} \\
\underbrace{\psi_{1}\left(x_{1}^{(1)}\right)-\psi_{1}\left(x_{1}^{(2)}\right)}_{\in \mathbb{N}}=\psi_{2}\left(x_{2}^{(2)}\right)-\psi_{2}\left(x_{2}^{(1)}\right)=\frac{1}{\partial} \underbrace{\left(y^{(2)}-y^{(1)}\right)}_{\in \mathbb{N}}
\end{gathered}
$$

If the node at a particular time slot attack strategy choice, the node will gain more profits, and the node will due to choose to attack, cause adverse effect to the whole network, make the forwarding probability of the other nodes, the benefit of the other nodes is also will be reduced. But because of the role of the punishment mechanism and the influence of discounted value, make the income gained by the attack on the node is eliminated, reduce the probability of attack node attack, so as to make the normal operation of the network. In the Figure 6, we illustrate the propsoed model.

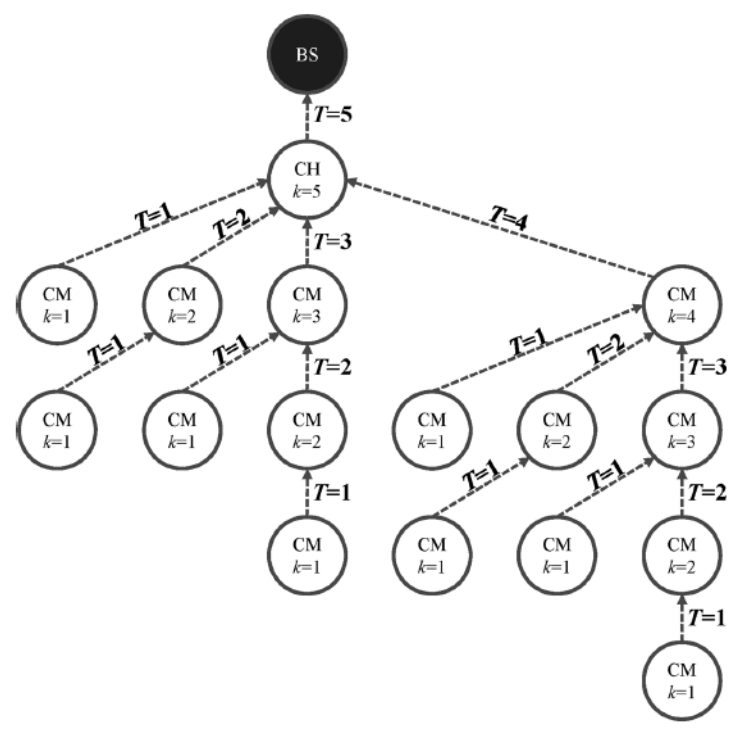

Figure 6. The Game Theory based Network Security and Robustness Enhancement Model 
Cluster is not only because of the burden of node scheduling management, data collection and the function such as task allocation, also need to burden collaborative interaction between cluster heads, data forwarding and other tasks, so the clustering topology's research focused on the load balancing cluster head load processing, namely network in local features, construct the clustering structure, the cluster head selection and reasonable distribution, the distribution of cluster members even, thus cluster load balance [29]. There are still some challenges we need to face to: (1) While enhance learning algorithm can learn package sent via regulating the learning cycle to reduce the number of nodes to save battery power, but the communication cost of node sends learning training package still is bigger, exploring how to more greatly save node resources remains a challenge, this paper puts forward the enhancement of learning can realize routing mechanism and combination of other technology in wireless sensor network in order to obtain a better effect, such as the node is not the optimal path is not as forwarding node, should make its dormant, to conserve energy. (2) Wireless sensor network security algorithm design of a limiting factor is the node of computing and storage capacity, distance jump Shared cross of key data authentication, nodes need to store the key information of the uplink and downlink connections, and before that we can want to store and use other node ID to generate the key information of key materials. After the discovery phase, nodes connected to compute its Shared key with the connection node. Message transmission phase, nodes is calculated using the Shared secret message authentication codes. Key distribution is the process of distribution and transfer the key is to use the password of the process of the relevant parties to receive the key. If it does not ensure safety use password parties to receive the keys will not be able to use if key cannot be delivered in time, will not be able to user password information system used for the guarantee of timely.

\section{Experimental Analysis and Simulation}

In this part, we conduct numerical analysis and simulation on the proposed methodology. Of the increase of sampling frequency adaptive adjustment adopts half way to adjust: when the sampling error is less than the preset threshold, halve each sensor in the clustering sampling frequency, or increase the sampling frequency, increase the step value of maximum threshold value and lower threshold. Finally limit adjusted sampling frequency in the upper threshold and lower threshold value range. In order to achieve the specific management functions, often need to spread in some specific area sensor collected data integration equipment. Administrators need to according to the management requirements, on the basis of the sensing device runtime model, select on behalf of the target data model fragment, this method can automatically generate the corresponding portfolio model, and support a portfolio model with a single runtime data synchronization of the model. Then, the administrator can in the form of combination model of target sensing device for unified management. In order to achieve different gradient cluster head load balancing, adopt the method of cluster weight more point, group of gradient nodes can have uniform organized into clusters, cluster load balance of the same gradient. To measure precision, the error of the prediction error using the average relative error index forecast to characterize the degree of deviation from the actual data. In the Figure 7 and Figure 8, we show the numerical analysis and simulation of the proposed method. 

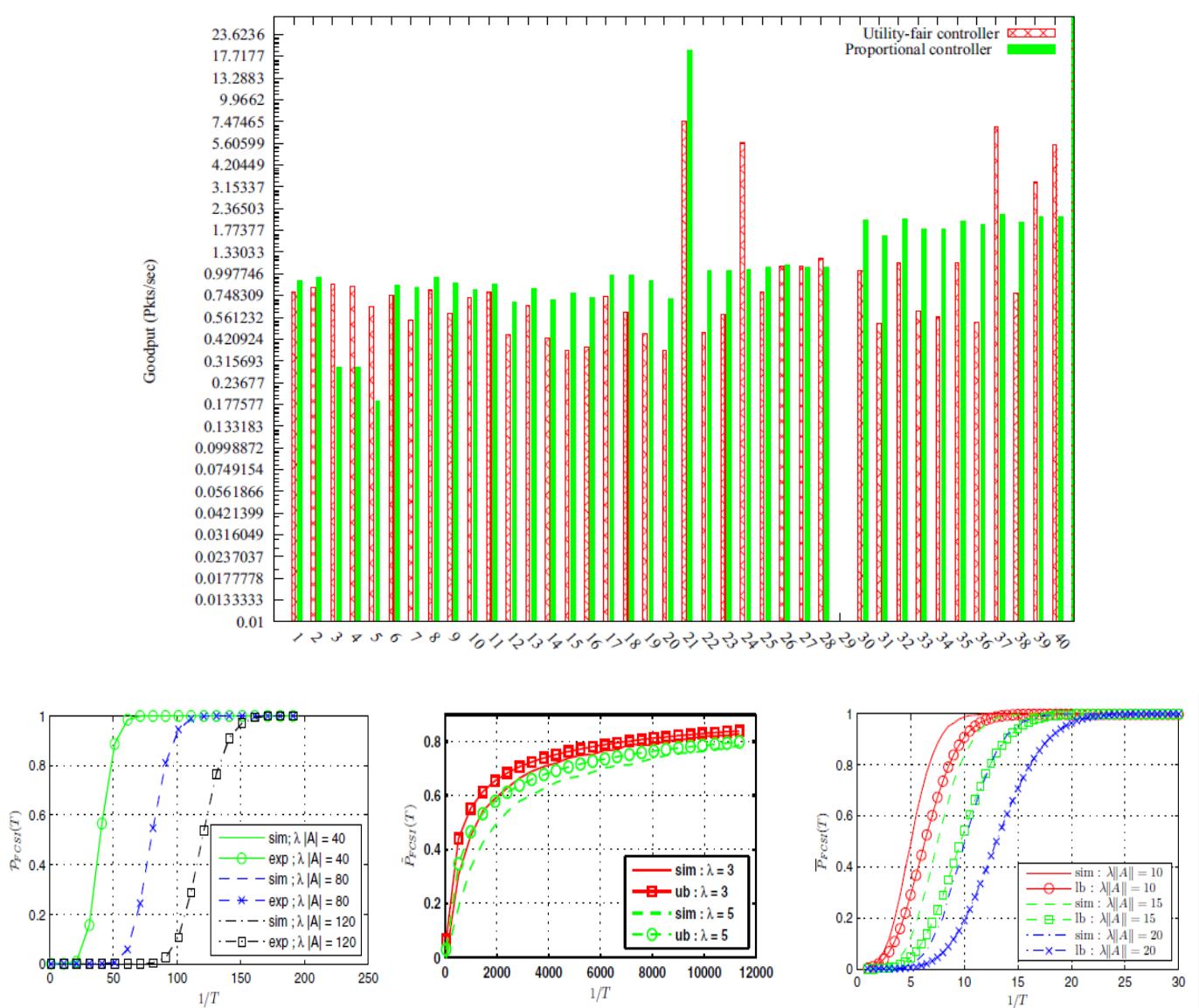

Figure 7. The Experimental Analysis and Result of the WSN Management

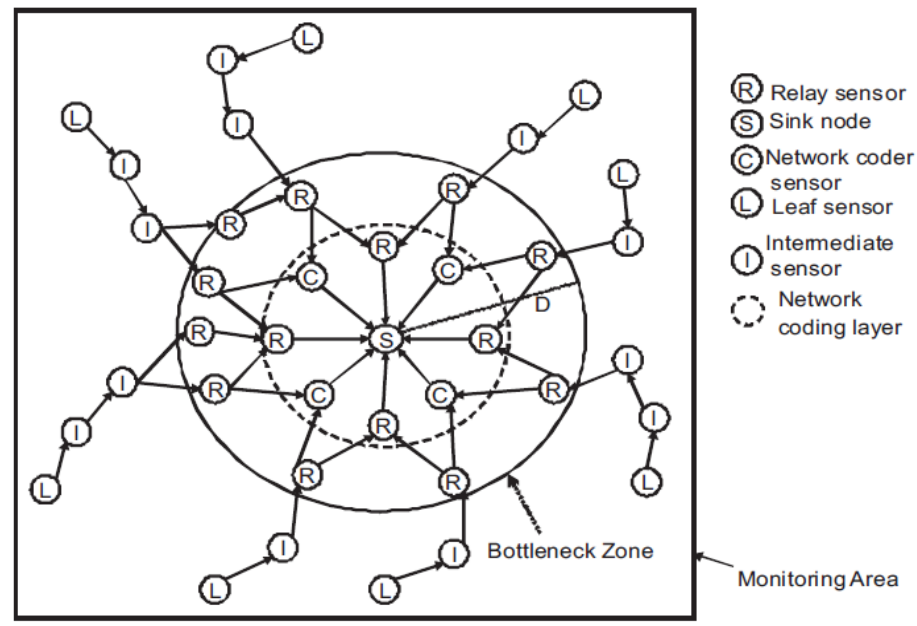

Figure 8. The Optimized Wireless Sensor Network Management Structure

\section{Conclusion and Summary}

In this paper, we conduct research on the wireless sensor network management methodologies based on the runtime model. We propose a kind of resources under the condition of limited support can be dynamically loaded wireless sensor network node middleware architecture. Detection and event boundary detection is one of the important applications of wireless sensor network and the node failure accurate detection is the precondition of improving the efficiency of events and event 
boundary detection. However, the current fault detection mechanism analysis of node failure type is not clear, causes the system could be mistaken for fault event boundary node, and often need for frequent communication between sensor nodes, utilization rate is low, lead to network system fault tolerant performance and node and additional energy costs. In the experimental part, we compare our method with other state-of-the-art algorithm and receive the positive feedback. In the future, we plan to conduct more related research to enhance the feasibility of the current approach.

\section{Acknowledgements}

This work is financially supported by the scientific research program of Shaanxi Provincial Education Department (Program No. 14JK2156).

\section{References}

[1] M.V. Ramesh, "Design, development, and deployment of a wireless sensor network for detection of landslides", Ad Hoc Networks, vol. 13, no. 1, (2014), pp. 2-18.

[2] H. Matsuura, "New routing framework for RPL: Constructing power-efficient wireless sensor network", Network Operations and Management Symposium (NOMS), 2014 IEEE, (2014), pp.1-9.

[3] M.C. Luculescu, S.C. Zamfira and L. Cristea, "Wisemans: wireless sensor network data management system for indoor climatic control", Mechanisms \& Machine Science, (2014).

[4] A.H. Kazmi, G.M.P. O'Hare, M.J. O'Grady, A.G. Ruzzelli and D.T. Delaney, "A review of wireless sensor network enabled building energy management systems", ACM Transactions on Sensor Networks, vol. 10, no. 4, (2014), pp. 136-144.

[5] B. Wang, Y. Zhang and X. Zhang, "Mixed key management scheme based on domain for wireless sensor network", Journal of Computer Applications, (2014).

[6] S.K. Sahoo and M.N. Sahoo, "An elliptic-curve-based hierarchical cluster key management in wireless sensor network", Advances in Intelligent Systems \& Computing, (2014), pp. 397-408.

[7] J. Wang, H. Wang, J. He, L. Li, M. Shen and X. Tan, "Wireless sensor network for real-time perishable food supply chain management", Computers \& Electronics in Agriculture, (2015), pp. 196-207.

[8] E. Tong, W. Niu, G. Li, D. Tang, L. Chang and Z. Shi, "Bloom filter-based workflow management to enable qos guarantee in wireless sensor networks", Journal of Network \& Computer Applications, vol. 39, no. 3, (2014), pp. 38-51.

[9] S. Kalantary and S. Taghipour, "A survey on architectures, protocols, applications, and management in wireless sensor networks [J]", Journal of Advanced Computer Science \& Technology, vol. 3, no. 1, (2014), pp. 1-11.

[10] S.C. Wang, S.S. Wang, C.W. Chen and K.Q. Yan, "A fuzzy-based management for power-aware wireless sensor network", Applied Mechanics \& Materials, (2014), pp. 479-480, 758-762.

[11] V. Jelicic, M. Magno, D. Brunelli, G. Paci and L. Benini, "Context-adaptive multimodal wireless sensor network for energy-efficient gas monitoring”, Sensors Journal, IEEE, vol. 13, no. 1, (2013), pp. 328338.

[12] M. Younis, I.F. Senturk, K. Akkaya, S. Lee and F. Senel, "Topology management techniques for tolerating node failures in wireless sensor networks: A survey", Computer Networks, vol. 58, (2014), pp. 254-283.

[13] M. Al Ameen, J. Liu and K. Kwak, "Security and privacy issues in wireless sensor networks for healthcare applications", Journal of medical systems, vol. 36, no. 1, (2012), pp. 93-101.

[14] Z. Li, X. Zhou, S. Li, G. Liu and K. Du, "Issues of wireless sensor network management", Lecture Notes in Computer Science, (2005), pp. 355-361.

[15] R. Tynan, D. Marsh, D. O'Kane and G.M.P. O'Hare, “Agents for wireless sensor network power management", Parallel Processing, 2005. ICPP 2005 Workshops. International Conference Workshops on IEEE, (2005), pp. 413-418.

[16] D. Marsh, D. O'Kane and G.M.P. O'Hare, "Agents for wireless sensor network power management", Parallel Processing, ICPP 2005 Workshops, International Conference Workshops on IEEE, (2005), pp. 413-418.

[17] J. Zhang and V. Varadharajan, "Wireless sensor network key management survey and taxonomy", Journal of Network \& Computer Applications, vol. 33, no. 2, (2010), pp. 63-75.

[18] T. Landstra, M. Zawodniok and S. Jagannathan, "Energy-Efficient Hybrid Key Management Protocol for Wireless Sensor Networks. Local Computer Networks", 2007, LCN 2007, 32nd IEEE Conference on, (2007), pp.1009-1016. 
[19] G. Tolle and D. Culler, "Design of an application-cooperative management system for wireless sensor networks", Wireless Sensor Networks, 2005. Proceeedings of the Second European Workshop on, (2005), pp.121-132, IEEE.

[20] C.H. Liu, C. Bisdikian, J.W. Branch and K.K. Leung, "Qoi-aware wireless sensor network management for dynamic multi-task operations", Sensor Mesh \& Ad Hoc Communications \& Networks Annual IEEE Communications Society, (2010), pp. 1-9.

[21] P. Hurni, M. Anwander, G. Wagenknecht, T. Staub and T. Braun, "TARWIS-A testbed management architecture for wireless sensor network testbeds", Network Operations and Management Symposium (NOMS), 2012 IEEE, vol. 131, (2012), pp. 611-614.

[22] S. Sana and M. Matsumoto, "A Wireless Sensor Network Protocol for Disaster Management", Information, Decision and Control, 2007. IDC '07, IEEE, (2007), pp. 209-213.

[23] C. Bi, Q. Zhang, R. Bao and H. Wang, "SAR image restoration and change detection based on game theory", In Intelligent Computing and Internet of Things (ICIT), 2014 International Conference on IEEE, (2015), pp. 55-58.

[24] P.H. Hsin and C.I. Chen, "Application of game theory on parameter optimization of the novel two-stage Nash nonlinear grey bernoulli model", Communications in Nonlinear Science \& Numerical Simulation, vol. 1, (2015), pp. 168-174.

[25] C. Hédoin, "Accounting for constitutive rules in game theory", Journal of Economic Methodology, (2015).

[26] U.F. Siddiqi, S.M. Sait and Y. Shiraishi, "A game theory-based heuristic for the two-dimensional VLSI global routing problem", Journal of Circuits Systems \& Computers, (2015).

[27] R.I.D. Silva, D.A.V. Del, A.M. Poersch and J.M.S. Nogueira, "Spatial query processing in wireless sensor network for disaster management”, Wireless Days (WD), 2009 2nd IFIP, IEEE, (2010), pp.1-5.

[28] B. Zhang and G. Li, "Survey of network management protocols in wireless sensor network", E-Business and Information System Security, 2009. EBISS '09. International Conference, (2009), pp. 1-5.

[29] S.A. Chaudhry, G. Boyle, W. Song and C. Sreenan, "EMP: A Network Management Protocol for IPbased Wireless Sensor Networks", Communication in Wireless Environments and Ubiquitous Systems: New Challenges (ICWUS), 2010 International Conference on IEEE, (2010), pp. 1-6.

Authors
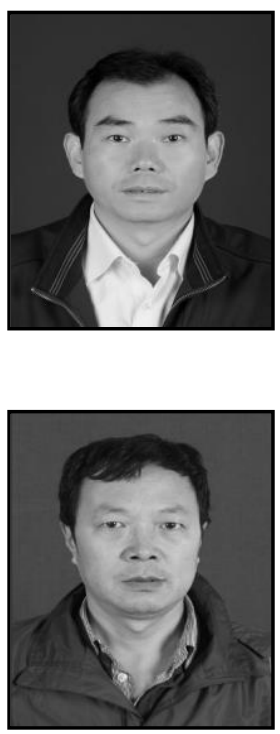

Wenzhun Huang, He received his B.S. and M.S. degrees in Communication and information system from Air Force Engineering University, China, in 1997. He received his Ph.D. degree in Information and communication engineering from Northwest Polytechnic University, China, in 2010. He is an associate professor in Xijing University. His research interests include information processing, anti-jamming technology, wireless communication system and IoT technology.

Shanwen Zhang, He received his B.S. degree in mathematics from Northwest University, China, in 1988. He received M.S. degree in applied mathematics from Northwest Polytechnic University, China, in 1995. He received Ph.D. degree in electromagnetic field and microwave from Air Force Engineering University, China, in 2001. He is a professor in Xijing University. His research interests include wavelet transforms, rough sets, genetic algorithm, pattern recognition and manifold learning. 
International Journal of Future Generation Communication and Networking Vol. 9, No. 3 (2016) 